\title{
Is the Calcium Pump Involved in Calcium Release?
}

\author{
Martina Ungeheuer, Andrea Migala, and Wilhelm Hasselbach \\ Max-Planck-Institut für Medizinische Forschung, Abteilung Physiologie, Jahnstraße 29, \\ D-6900 Heidelberg, Bundesrepublik Deutschland \\ Z. Naturforsch. 41c, 647-651 (1986); received March 18, 1986 \\ Sarcoplasmic Reticulum, Calcium Release, Caffeine, Thiol Blockage \\ The blockage of all thiol residues accessible to the mercurial mersalyl in the sarcoplasmic \\ reticulum membranes resulting in complete inactivation of the membranes' calcium transport \\ system does interfere neither with caffeine- nor calcium-induced calcium release from actively \\ loaded membrane vesicles.
}

\section{Introduction}

The contractile systems of smooth and skeletal muscles are activated by the release of calcium ions from their intracellular stores in the cisternal elements of the sarcoplasmic reticulum [1,2]. Contractile activity is turned off after the calcium pump in the reticulum has removed calcium from the contractile proteins and a resting calcium level of $0.1 \mu \mathrm{M}$ has been reached ( $c f$. [3]). While most of the physiologically important features of the mechanism by which the calcium pump operates are known, our concept concerning the mechanism of calcium release has remained quite incomplete. That is because the mechanism of calcium release in the living muscle is difficult to analyse due to the limited possibilities to selectively manipulate the release mechanism. In skeletal muscle a close correlation between calcium release and the movement of charges in the plasma membrane or its T-tubular invaginations could be established suggesting that the electrical event in the plasma membrane might trigger calcium release [4]. Permeabilized muscle cells and fibres allow larger variation of the experimental conditions but are hampered by the interfering activity of the contractile proteins. These preparations allow to study the effect of various drugs on the release mechanism, such as caffeine, quercetin, calcium ions etc. [5-7]. The effect of calcium itself as a releasing agent has intensively been studied by A. Fabiato [8] in surviving muscle fibres. These studies were prompted by the findings of Endo [9], who, under special conditions, could induce calcium release from isolated cal-

Abbreviation: NEM, N-ethylmaleimide.

Reprint requests to Prof. W. Hasselbach.

Verlag der Zeitschrift für Naturforschung, D-7400 Tübingen $0341-0382 / 86 / 0500-0647 \$ 01.30 / 0$ cium loaded sarcoplasmic reticulum vesicles by the addition of calcium ions. The biochemical approach to study calcium release from isolated sarcoplasmic reticulum membranes has led to the definition of the experimental conditions, under which calcium release can occur. There seems to be general agreement that calcium release from actively and passively loaded sarcoplasmic reticulum vesicles can be induced by the application of various drugs, by suddenly elevating the calcium level in the external medium or by changing the ionic conditions, i.e. by introducing an additional permeant ion [10-14]. Yet, contradicting results were reported as to the distribution of the calcium releasing elements in the sarcoplasmic reticulum and their sensitivity towards the various releasing agents. These contradictions might be related to the repeatedly suggested involvement of the calcium transport protein in the release mechanism (cf. [13]).

This report will focus on the possible role of the calcium transport protein in the mechanism of calcium release. We rapidly inactivated the calcium transport enzyme by blocking its essential thiol groups with the fast reacting mercurial - mersalyl and studied the effect of this manipulation on the caffeine and calcium induced calcium release. The results argue against an involvement of the calcium pump in the release mechanism.

\section{Materials and Methods}

A heavy fraction of sarcoplasmic vesicles was prepared from rabbit skeletal muscle as described by $\mathrm{Su}$ and Hasselbach [10]. Active calcium loading in the absence of calcium precipitating anions was performed in media containing ATP, supplemented by phosphoenol pyruvate and pyruvate kinase as ATP 
regenerating system. Calcium uptake and release assay contained: $0.2 \mathrm{mg}$ protein $\cdot \mathrm{ml}^{-1}, 20 \mu \mathrm{M}{ }^{45} \mathrm{Cl}_{2}$, $2 \mathrm{~mm}$ ATP, $3 \mathrm{~mm} \mathrm{MgCl}_{2}, 4 \mathrm{~mm}$ phosphoenol pyruvate, $0.04 \mathrm{mg} \cdot \mathrm{ml}^{-1}$ pyruvate kinase, $100 \mathrm{~mm} \mathrm{KCl}$, $20 \mathrm{~mm}$ histidine, $\mathrm{pH} 7.0$, at $20^{\circ} \mathrm{C}+/-2{ }^{\circ} \mathrm{C}$.

Mersalyl was added from freshly prepared stock solutions in $0.1 \mathrm{M} \mathrm{KCl}$. Calcium release was measured at appropriate time intervals after either mersalyl applications (control), addition of caffeine alone (second control) or after successive additions of mersalyl and caffeine. For inducing calcium release by calcium ions instead of caffeine, calcium EGTA solutions were added giving a final free calcium concentration in the assay of $3 \mu \mathrm{M}$. Calcium uptake and release were terminated by millipore filtration. The radioactivity in filtrate was determined by liquid scintillation counting. ATP hydrolysis was measured in the calcium uptake medium as previously described [15]. In the presence of caffeine ${ }^{32} \mathrm{P}-\gamma$ ATP was used to determine ATPase activity. ${ }^{32} \mathrm{P}_{\mathrm{i}}$ was extracted according to Avron [16]. ${ }^{32} \mathrm{P}$-radioactivity was measured by liquid scintillation counting. $\mathrm{Na}_{2}$ ATP, phosphoenol pyruvate and pyruvate kinase were obtained from Boehring Mannheim (FRG). Mersalyl acid was purchased from Sigma, St. Louis (USA).

\section{Results}

A most characteristic feature of the sarcoplasmic reticulum transport system is the simultaneous but irreversible blockage of calcium-dependent ATP hydrolysis and calcium transport activity by thiol reagents $[15,18,19]$. When four of the twenty one thiol residues of the transport enzyme are substituted by alkylating agents like NEM or mercurials like mersalyl, both activities are completely abolished. In contrast to the calcium-dependent ATPase, the calcium-independent ATPase which contaminates all native vesicular preparations, is not affected by thiol reagents (Fig. 1A). Thus, on addition of a thiol reagent, ATP hydrolysis declines from the high rate of calcium dependent to the lower rate of calcium independent activity. In general, mercurials effect inactivation much more rapidly than alkylating agents. We therefore applied mersalyl for pump inactivation. Mersalyl at a concentration of $0.1 \mathrm{~mm}$ added to $0.2 \mathrm{mg} / \mathrm{ml}$ completely inactivates the enzyme in a few seconds. However, when mersalyl is added to the complete ATP containing assay, inhibi- tion develops more slowly (Fig. 1B). This is in agreement with the protective effect exerted by ATP which has been reported to considerably retard the substitution of 1 to 2 thiol residues belonging to the class of thiol groups which slowly react with 5,5'dithiobis(2-nitrobenzoate) [18, 19]. As shown in Fig. 1B, complete inhibition of the calcium activated enzyme requires a reaction time of $1 \mathrm{~min}$, after which the ATP is cleaved by the calcium-indepen-
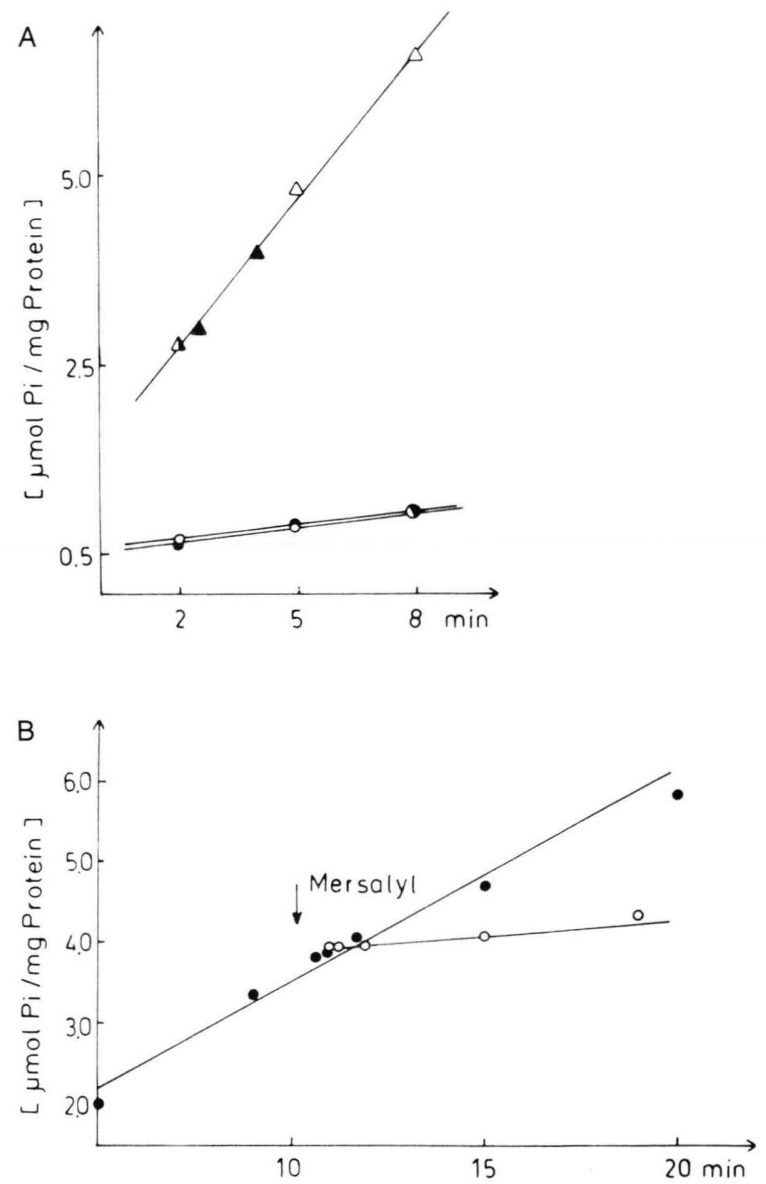

Fig. 1. ATP hydrolysis of calcium-loaded vesicles and its inhibition by mersalyl.

(A) ATP hydrolysis in the presence of $10 \mu \mathrm{M}$ A28137. Calcium-activated ATPase in the absence $(\triangle)$ and in the presence of $10 \mathrm{~mm}$ caffeine ( $\mathbf{\Delta})$. ATP hydrolysis in the presence of 1 mм EGTA (-); ATP hydrolysis in calcium containing uptake medium in the presence of $0.1 \mathrm{~mm}$ mersalyl $(\bigcirc)$.

(B) The vesicles were actively loaded with calcium as described in Materials and Methods. Aliquots were taken for phosphate determination at times given on the abscissa.

(-) Progress of phosphate liberation after the cessation of net calcium uptake. (O) Phosphate liberation after the addition of mersalyl $(0.1 \mathrm{~mm})$. 
dent ATPase only. When mersalyl is added to sarcoplasmic reticulum vesicles which have actively been loaded with calcium in the absence of precipitating agents, the vesicle starts to loose calcium ions not before the enzyme is completely inactivated (Figs. 2 and 3). Subsequently, calcium release progressively rises with a half time of $2.5 \mathrm{~min}$. At the given calcium load of $95 \mathrm{nmol} / \mathrm{mg}$ the mersalyl-induced release is moderately accelerated as compared to passive calcium efflux from native vesicles affected by EGTA [20]. Hence, it is possible by the application of mersalyl, to completely block the pump at moderate alteration of the membrane permeability for at least $1 \mathrm{~min}$ following mersalyl addition. After complete abolition of the pump by mersalyl, this time interval allows us to check if the action of specific calcium releasing agents such as caffeine or calcium ions requires an intact calcium pump. As illustrated on addition of $10 \mathrm{~mm}$ caffeine (Fig. 2) or by suddenly increasing the free calcium concentration in the solution (Fig. 3), calcium is as rapidly released from mersalyl-poisoned as from native preparations. No measurable effect of mersalyl on the amplitude of release can be detected. $25 \mathrm{nmol}$ and $50 \mathrm{nmol} / \mathrm{mg}$ calcium are released by caffeine and calcium respectively from native and mersalyl treated preparations. Due to the inhibition of the pump, however, no re-uptake of calcium occurs. This finding implies that an operating calcium pump is required neither for the caffeine nor for the calcium-induced calcium release and furthermore, that

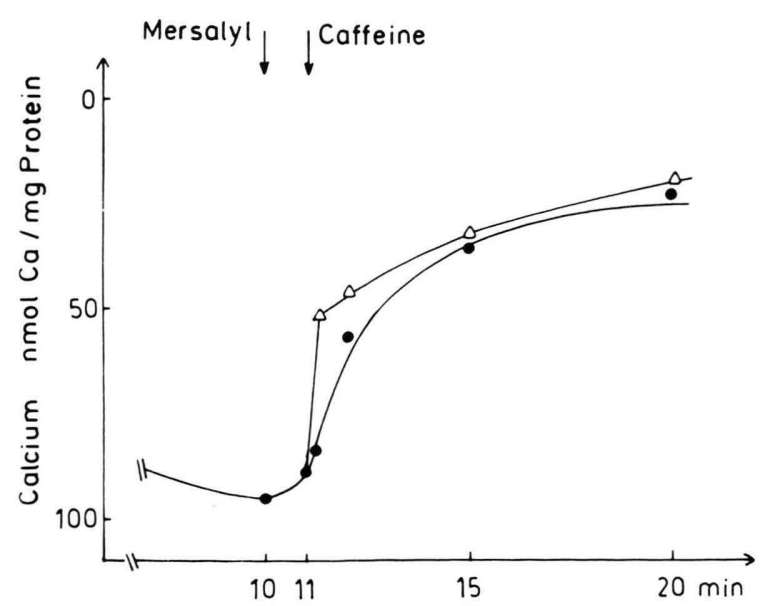

Fig. 2. Caffeine-induced calcium release after blocking the enzyme's thiol residues with mersalyl. After calcium loading $0.1 \mathrm{~mm}$ mersalyl (final concentration) was added (๑). $1 \mathrm{~min}$ after the addition of mersalyl $10 \mathrm{~mm}$ caffeine were applied $(\triangle)$.

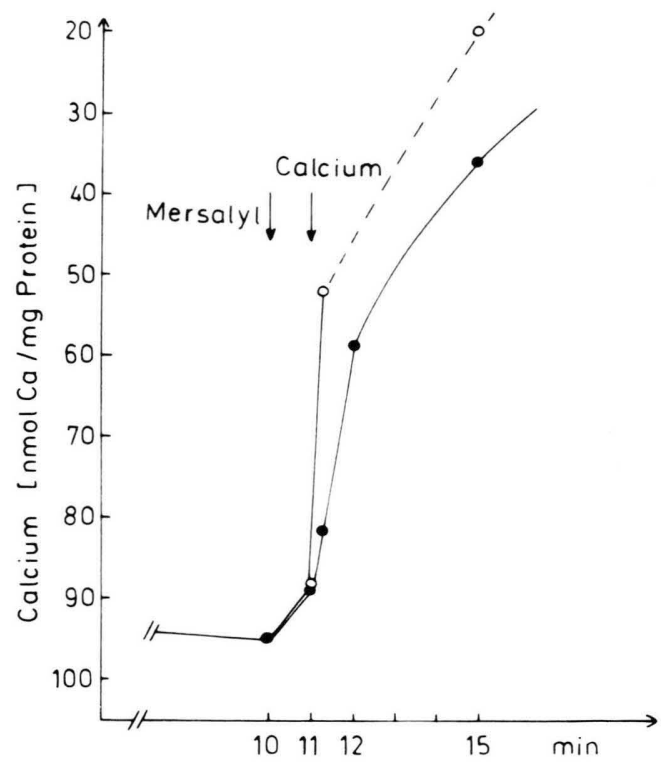

Fig. 3. Calcium-induced calcium release after blockage of the enzyme thiol groups with mersalyl. After calcium loading $0.1 \mathrm{~mm}$ mersalyl was added (final concentration) (๑). $0.2 \mathrm{~mm}$ calcium $/ 0.3 \mathrm{~mm}$ EGTA was added $1 \mathrm{~min}$ after mersalyl addition (O).

the proteins which constitute the calcium releasing structures, the calcium channels, do not contain mersalyl sensitive thiol residues.

\section{Discussion}

The results obtained in this study, showing that the caffeine-induced as well as the calcium-induced release of calcium from actively loaded sarcoplasmic reticulum vesicles is not affected when the calcium pump is blocked, clearly indicate that an intact calcium pump is not required for rapid calcium release. An involvement of the pump in the mechanism of calcium release has repeatedly been discussed in the past. The release of calcium produced by caffeine, quercetin and some other reagents was assumed to be brought about by a supposed effect of the drugs on pump activity. None of the drug effects, however, can be related to pump inhibition only, because pump inhibition should only lead to an uncompensated calcium efflux. The rate of this unidirectional calcium movement evaluated by flux measurement under steady state conditions as well as observed in this and previous studies is much smaller than the rates observed for the drug-induced release [21]. The 
calcium concentrations which effect calcium release in our experiments very well agree with the calcium concentrations reported by Fabiato [8] for calciuminduced calcium release in living fibres. The affinities of calcium for the releasing sites referred to are difficult to compare with the data obtained by Meissner [14] and Nagasaki and Kasai [22] for passively loaded preparations, because these studies were performed in the absence of magnesium ions. The presence of distinct releasing sites other than the pump protein is supported by Meissner's [14] observation showing that calcium-induced calcium release is confined to a heavy membrane fraction and by the findings of $\mathrm{Su}$ and Hasselbach [10] indicating that only a subpopulation of the heavy membrane fraction is caffeinesensitive. However, these findings appear to be in conflict with the observation of Ikemoto [11] who reported caffeine and calcium-induced calcium release from light membrane fractions obtained by French Press treatment of the preparations. The observation reported in the accompanying paper that caffeine and calcium sensitive membrane preparations can be made insensitive while maintaining their pump activity shows that it is certainly not the pump alone which conveys calcium and caffeine sensitivity directly. The discussed discrepancy can be explained by the different procedures applied for obtaining Ttubular-free membranes. Meissner's procedure [14] of differential pelleting and the procedure applied by Su and Hasselbach [10] are more gentle than French Press treatment. The caffeine and calcium sensitive constituents which might originally be confined to the triad region of the sarcoplasmic reticulum would be distributed over all components of the reticulum during French Press treatment.

Neither the isolation of a caffeine- and calciuminsensitive membrane fraction nor the desensitiza- tion of previously sensitive preparations do completely rule out an indirect role of the pump protein as transducing elements for specified calcium channel structures. Such a transducing function of the pump would be in agreement with the fact that calcium release and pump function are activated in the same calcium concentration range. The indirectly induced release is at variance with the well-supported two-stage-mechanism of calcium transport [23]. According to this mechanism the formation of a high calcium gradient by the vesicles should result in the transformation of external high into internal low affinity calcium binding sites. Hence under these conditions calcium ions applied to the external medium should not meet high affinity calcium binding sites required for calcium release ( $c f$. [24]). It is also not very likely that caffeine, the effect of which was also found to be mersalyl-resistant, primarily interacts with the calcium pump, since caffeine does not interfere with calcium pump activity (Fig. 1A). Our result that thiol group blockage does not induce a rapid calcium release in amounts comparable to the number of the pump's high affinity sites, is in line with the fact that the high affinity calcium binding sites in the pump molecule are located in an aminoacid sequence devoid of cystein residues [25]. The absence of cystein residues in the calcium binding regions, on the other hand, is difficult to reconcile with the calcium release effected by a number of heavy metals supposed to react with sulphydril groups [26]. Yet, part of this release might be attributed to a calcium displacement from its anionic binding sites in the membrane by the heavy metal ions. The reported permeability increase effected by these agents might result from the severe structural changes which slowly occur subsequent to extensive thiol substitution [18]. 
[1] J. R. Blinks, R. Rüdel, and S. R. Taylor, J. Physiol. 277, 291-323 (1978).

[2] A. V. Somlyo, H. Gonzales-Serrato, H. Shunan, G. McClellan, and A. P. Somlyo, J. Cell Biol. 90, 577-594 (1981).

[3] W. Hasselbach and H. Oetliker, Am. Rev. Physiol. 45, 125-139 (1983).

[4] W. Melzer, M. F. Schneider, B. J. Simon, and G. Szues, J. Physiol. 373, 481-511 (1986).

[5] J. Su and D. A. Malencik, Pflügers Arch. 394, 48-54 (1982).

[6] E. W. Stephenson, J. Gen. Physiol. 86, 833-852 (1985).

[7] V. Shoshan, K. P. Campbell, D. H. MacLennan, W. Frodis, and B. Britt, Proc. Nat. Acad. Sci. USA 77, 4435-4438 (1980).

[8] A. Fabiato, J. Gen. Physiol. 85, 291-320 (1985).

[9] M. Endo, M. Tanaka, and Y. Ogawa, Nature 228, 34-36 (1970).

[10] J. Su and W. Hasselbach, Pflügers Arch. 400, 14-21 (1984).

[11] N. Ikemoto, A. Bozena, and D. H. Kim, J. Biol. Chem. 259, 13151-13158 (1984).

[12] N. Ikemoto, A. Bozena, and L. G. Mészáros, J. Biol. Chem. 260, 14096-14100 (1985).

[13] M. Chiesi and Y. S. Wen, J. Biol. Chem. 258, 6078-6085 (1983).
[14] G. Meissner, J. Biol. Chem. 259, 2365-2374 (1985).

[15] W. Hasselbach and M. Makinose, Biochem. Z. 333, 518-528 (1961)

[16] M. Avron, Biochim. Biophys. Acta 40, 257-272 (1960).

[17] M. Makinose and W. Hasselbach, Biochem. Z. 343, 360-382 (1965).

[18] W. Hasselbach and K. Seraydarian, Biochem. Z. 345, 195-172 (1966).

[19] D. A. Thorley-Lawson and N. M. Green, Biochem. J. 167, 739-748 (1977).

[20] J. J. Feher and I. N. Briggs, J. Biol. Chem. 257, 10191-10199 (1982).

[21] W. Waas and W. Hasselbach, Europ. J. Biochem. 116, 601-608 (1981).

[22] K. Nagasaki and M. Kasai, J. Biochem. (Tokyo) 90, $749-755$ (1981)

[23] L. de Meis, The Sarcoplasmic Reticulum in Transport, in: Life Science (E. E. Bittar, ed.), pp. 1-168, John Wiley Sons, New York 1981.

[24] P. Medda and W. Hasselbach, Europ. J. Biochem. 137, 7-14 (1983).

[25] D. H. MacLennan, C. J. Brandl, B. Korczak, and N. M. Green, Nature 316, 696-700 (1985).

[26] J. Abramson, J. Trim, L. Wedan, and G. Salma, Proc. Natl. Acad. Sci. USA 80, 1526-1530 (1983). 